\section{Analysis of Patient Handoff Between Providers at a Tertiary Urban Medical Center}

Candice Metzinger, MS-21, Sam Antonios, M.D., ${ }^{2,3}$, K. James Kallail, Ph.D. ${ }^{3,4}$, Hayrettin Okut, Ph.D. , Rosey Zackula, M.A., Brianna Cline, D.O. ${ }^{3}$

${ }^{1}$ University of Kansas School of Medicine-Wichita, Wichita, KS

${ }^{2}$ Ascension Via Christi St. Francis, Wichita, KS

University of Kansas School of Medicine-Wichita, Wichita, KS

${ }^{3}$ Department of Internal Medicine

${ }^{4}$ Office of Research

Received Feb. 7, 2021; Accepted for publication April 14, 2021; Published online Aug. 4, 2021 https: /oi.org $10.17161 /$ kjm.vol1415170

\section{ABSTRACT}

Introduction. Few studies have quantified the total number of attending and consulting physicians involved in inpatients' care, and no other research quantifies the total number of all providers participating in inpatients' care. The purpose of this study was to calculate the number of attending hand-offs, the attending encounter time, and the total number of providers participating in inpatients' care for all admitted patients at a tertiary urban medical center.

Methods. The study design was an observational retrospective cohort. Subjects included pediatric and adult patients who were admitted to and discharged from Ascension Via Christi St. Francis (AVCSF) in Wichita, Kansas between November 1, 2019 and January 31, 2020. Data were abstracted from the Cerner Electronic Medical Record. Variables included: patient demographics, admitting diagnosis, diagnosis related group (DRG), admission service, and duration of inpatient stay. Provider variables abstracted included provider type and provider specialty. Categorical variables were presented as frequencies and percentages, while continuous variables were presented as means \pm standard deviation.

Results. The sample included information from 200 patient charts. Patients' ages ranged from 5 to 94 years, with a mean of 61 years. Approximately $52 \%$ were female and $74.9 \%$ were admitted to a surgical service. The length of all inpatients' stays ranged from less than 1 day to 31 days, with a mean of 4 days. Seventy-six different DRGs were recorded. The most frequent attending specialties were hospital medicine, internal medicine, general surgery, and interventional cardiology. Consulting physicians had more patient encounters than any other healthcare provider. For all inpatients, an average of two attending physicians participated in care over the duration of their stay with a range of one to six attending physicians. There was an average of one hand-off between attending physicians. Patients had an average of five consulting physicians, two resident physicians, two physician assistants, and two nurse practitioners during a stay. There was an average of 10 total providers, with a range of one to 46 total providers participating in care.

Conclusions. Understanding the provider data surrounding an inpatient stay is a foundational step in assessing the quality of the provider-inpatient encounter and potential areas for improvement. In this study, the average number of attending physicians and handoffs was reasonable; however, the total number of providers involved in care was relatively high. Assessment of staffing and scheduling requirements by This is an open-access article distributed under the terms of the Creative Commons Attribution Nonorg/licenses/by-nc-nd/4.0/) hospital administration could identify areas of improvement to reduce the potential for medical error caused by multiple providers being involved in patient care. Kans J Med 2021;14:192-196

\section{INTRODUCTION}

During an inpatient stay, more than one healthcare provider participates in patient care. ${ }^{1}$ Collectively, these providers form a multidisciplinary healthcare team, ${ }^{2}$ lead by the attending physician. This provider is ultimately responsible for the diagnosis, treatment, and management of the patient. Consulting physicians (sometimes referred to as specialists) are those who are highly trained in a specific area of medicine and are utilized by attending physicians to help with diagnosis and treatment of the patient. Resident physicians are licensed physicians participating in further specialty training after completing medical school. Advanced practice professional providers, such as nurse practitioners and physician assistants, have advanced training and assist physicians in the management of patients.

Over the course of an inpatient stay, attending physicians may transfer, or hand-off, care to another physician who assumes the attending physician role. Increased frequencies of hand-offs may lead to medical errors and/or adverse events. ${ }^{3}$ The primary causes for these errors and adverse events are low quality hand-off procedures and multiple physicians participating in inpatients' care. The quality and content of patient hand-offs has been studied, especially in emergency departments (ED) and intensive care units (ICU); ${ }^{3,5}$ however, there is minimal research investigating the number of attending hand-offs, the length of time an attending is responsible for a patient (defined as attending encounter time), and the total number of providers (physicians and advanced practice professional providers) participating in a patients' care during a hospitalization. ${ }^{4}$

Although some research described the impact of the number of attending physicians on inpatients' outcomes and satisfaction, ${ }^{6}$ few quantified the total number of attending and consulting physicians involved in inpatients' care, and no other research quantified the total number of providers participating in inpatients' care, including attending physicians, consulting physicians, resident physicians, physician assistants, and nurse practitioners. Quantitative analysis of attending encounter time, which has the potential to impact the number of attending hand-offs, also is lacking. The purpose of this study was to calculate the number of attending hand-offs, the attending encounter time, and the total number of providers participating in inpatients' care for all admitted patients at a tertiary urban medical center.

\section{METHODS}

The study design was an observational retrospective cohort. The study was approved by the Institutional Review Board at Ascension Via Christi.

Participants. Pediatric and adult patients who were admitted to and discharged from Ascension Via Christi St. Francis (AVCSF) in Wichita, Kansas between November 1,2019 and January 31,2020 were included in this study. Those who were admitted for labor and delivery, including 


\section{KANSAS JOURNAL of MEDICINE}

PATIENT HANDOFFS

continued.

the infants born during the admission, were excluded.

Procedures. Data were abstracted from the hospital's electronic medical record. The abstracted variables included: patient demographics (age and sex), admitting diagnosis (ICD-10), diagnosis related group (DRG), admission service (medical or surgical), and duration of inpatient stay (in days). Abstracted provider variables included provider type (e.g., attending physician, nurse practitioner) and provider specialty (e.g., hospitalist, diagnostic radiology, general surgery).

Duration of care was abstracted for attending physicians only. These data were calculated by subtracting intake and hand-off dates. Intake date (defined as the date and time the attending physician assumed responsibility for the patient) was identified in two ways. The admitting physician was identified as the first attending physician, so the admission date and time were recorded as the intake date. If an attending physician was not the admitting physician, the intake date was recorded as the date and time of the provider's first documentation in the patient chart. The date and time recorded as the second attending physician's intake date also was used as the hand-off date for the first attending physician. Patient discharge date and time was recorded as the handoff date for the last attending physician to document in the chart prior to discharge.

Any repeat attending physicians (i.e., attending physicians who participated in patient care for two non-contiguous periods during the patient stay) were flagged in the database to ensure the repeat instance was subtracted from the total number of providers. All other providers (e.g., consulting physicians, resident physicians, physician assistants, and nurse practitioners) were recorded once, whether or not they had multiple encounters with the patient.

Statistical Analysis. Sample size was determined through a power analysis using summary statistics for 32,342 inpatient discharges over one fiscal year. The length of stay from the summary statistics was assumed to have a lognormal distribution, with the mean length of stay and the coefficient of variation used to estimate sample sizes for different power levels. From these calculations, a power of 0.938 for a sample of 200 patients resulted. A report of all patients admitted and discharged during the study timeframe was produced. A data report generated by AVCSF containing a random sample of 250 patients from the study timeframe was obtained, and the first 200 patients were entered into a REDCap ${ }^{\oplus}$ database. ${ }^{7}$ The remaining 50 patients in the report were not included in the initial chart abstraction and were kept as backup in the event that any of the initial 200 patients were excluded from the study. Data were abstracted from patient charts, and REDCap ${ }^{\oplus}$ was used to calculate age at admission, length of stay, attending encounter time, and the number of providers per inpatient stay.

De-identified data were exported from REDCap into SAS version 9.4 for statistical analysis. Categorical variables were presented as frequencies and percentages, while continuous variables were presented as means \pm standard deviation (SD). A 2 -sided p value of less than 0.05 was considered statistically significant. Statistical analysis was con-

\section{RESULTS}

Of the initial 200 patient charts that were reviewed, 5.5\% (n = 11) were omitted due to admission dates that were prior to the study timeframe. To satisfy the power requirement for statistical significance, 11 additional patient charts from the original randomized report were added to the data abstraction, and the final sample consisted of 200 patient charts.

Patient Characteristics. Patients' ages ranged from 5 to 94 years, with a mean of 61 years $(\mathrm{SD}=15.3)$. Approximately $52 \%(\mathrm{n}=103)$ were female and most $(74.9 \%, \mathrm{n}=158)$ were admitted to a surgical service. When subdivided by admission service, average age was similar to the overall average for medical and surgical patients (62 years and 61 years, respectively). The majority of patients admitted to the surgical service were female $(53.8 \%, \mathrm{n}=85)$ and the majority of patients admitted to the medical service were male $(57.1 \%, \mathrm{n}=24)$. The length of all inpatients' stays ranged from less than 1 day to 31 days, with a mean of four days ( $\mathrm{SD}=3.75)$. Patients admitted to the medical service had a mean stay of five days ( $\mathrm{SD}=3.85)$ and those admitted to the surgical service had a mean stay of four days $(\mathrm{SD}=3.75)$.

There were 76 different Diagnosis Related Groups (DRGs) recorded for this sample. The most frequent for all patients were "Major Hip and Knee Joint Replacement or Reattachment of Lower Extremity without Major Complication or Comorbidity" $(12.0 \%, \mathrm{n}=24)$, "O.R. Procedures for Obesity without Complication or Comorbidity/Major Complication or Comorbidity" (11.5\%, n = 23), "Major Small \& Large Bowel Procedures with Complication or Comorbidity" $(8.0 \%, \mathrm{n}=$ 16), and "Major Small \& Large Bowel Procedures without Complication or Comorbidity/Major Complication or Comorbidity” (5.0\%, $\mathrm{n}=10$ ). When subdivided by admission service, patients admitted to the surgical service had the same most frequent DRGs as the total patient population. The most frequent DRGs for patients admitted to the medical service were "Seizures without Major Complication or Comorbidity" (14.3\%, n=6), "Percutaneous Cardiovascular Procedure with Drug Eluting Stent without Major Complication or Comorbidity" $(11.9 \%, \mathrm{n}=5)$, and "Septicemia or Severe Sepsis without Mechanical Ventilation Greater than 96 Hours" $(11.6 \%, \mathrm{n}=5)$.

When all DRGs were categorized by Major Diagnostic Categories (MDC), the most frequent were "Diseases \& Disorders of the Musculoskeletal System \& Connective Tissue" (31.0\%, n = 61), "Diseases \& Disorders of the Digestive System" (18.0\%, $\mathrm{n}=35)$, "Diseases \& Disorders of the Circulatory System" (15.0\%, $\mathrm{n}=30)$, and "Endocrine, Nutritional \& Metabolic Diseases \& Disorders" (15.0\%, $\mathrm{n}=30$; Table 1).

Table 1. Major diagnostic categories for all patients.

\begin{tabular}{|l|c|c|}
\hline Major diagnostic categories & Frequency & Percent \\
\hline $\begin{array}{l}\text { O8 Diseases \& Disorders of the Musculoskeletal } \\
\text { System \& Connective Tissue }\end{array}$ & 61 & $30.5 \%$ \\
\hline 06 Diseases \& Disorders of the Digestive System & 35 & $17.5 \%$ \\
\hline 05 Diseases \& Disorders of the Circulatory System & 30 & $15.0 \%$ \\
\hline $\begin{array}{l}\text { 10 Endocrine, Nutritional \& Metabolic Diseases \& } \\
\text { Disorders }\end{array}$ & 30 & $15.0 \%$ \\
\hline O1 Diseases \& Disorders of the Nervous System & 14 & $7.0 \%$ \\
\hline All other & 30 & $15.0 \%$ \\
\hline
\end{tabular}


Provider Characteristics. Attending physicians comprised 15.0\% $(\mathrm{n}=304)$ of all provider types (Table 2$)$. The most frequent attending specialties for medical patients were hospital medicine $(62.0 \%, \mathrm{n}=$ 49), internal medicine ( $11.4 \%, \mathrm{n}=9)$, general surgery $(8.9 \%, \mathrm{n}=7)$, and interventional cardiology $(7.6 \%, \mathrm{n}=6)$. The most frequent attending specialties for surgical patients were general surgery $(42.9 \%, \mathrm{n}=82)$, orthopedic surgery $(23.0 \%, \mathrm{n}=44)$, cardiothoracic surgery $(11.0 \%, \mathrm{n}=$ $21)$, and neurosurgery $(9.4 \%, \mathrm{n}=18$; Table 3$)$.

Table 2. Provider type for all patients.

\begin{tabular}{|l|c|c|}
\hline Provider type & Frequency & Percent \\
\hline Attending Physician & 304 & $15.0 \%$ \\
\hline Consulting Physician & 952 & $46.9 \%$ \\
\hline Resident Physician & 291 & $14.3 \%$ \\
\hline Physician Assistant & 150 & $7.4 \%$ \\
\hline Nurse Practitioner & 333 & $16.4 \%$ \\
\hline
\end{tabular}

Table 3. Attending specialty by service.

\begin{tabular}{|c|c|c|}
\hline Type of service & Frequency & Percent \\
\hline \multicolumn{3}{|l|}{ Medical } \\
\hline Hospitalist & 49 & $62.0 \%$ \\
\hline Internal Medicine & 9 & $11.4 \%$ \\
\hline General Surgery & 7 & $8.9 \%$ \\
\hline Interventional Cardiology & 6 & $7.6 \%$ \\
\hline Cardiology & 4 & $5.1 \%$ \\
\hline Neuro Critical Care & 2 & $2.5 \%$ \\
\hline Orthopedic Surgery & 1 & $1.3 \%$ \\
\hline Pediatrics & 1 & $1.3 \%$ \\
\hline \multicolumn{3}{|l|}{ Surgical } \\
\hline General Surgery & 82 & $42.9 \%$ \\
\hline Orthopedic Surgery & 44 & $23.0 \%$ \\
\hline Cardiothoracic Surgery & 21 & $11.0 \%$ \\
\hline Neurosurgery & 18 & $9.4 \%$ \\
\hline Interventional Cardiology & 11 & $5.8 \%$ \\
\hline Vascular Surgery & 10 & $5.2 \%$ \\
\hline Plastic Surgery & 2 & $1.0 \%$ \\
\hline Urology & 2 & $1.0 \%$ \\
\hline Cardiology & 1 & $0.5 \%$ \\
\hline
\end{tabular}

Consulting physicians had more patient encounters than any other healthcare provider, comprising $46.9 \%(n=952)$ of all providers in the study (Table 2). The most frequent consultant specialties for medical patients were nephrology $(9.2 \%, \mathrm{n}=20)$, cardiology $(8.3 \%, \mathrm{n}=18)$, and pulmonary disease $(5.0 \%, \mathrm{n}=11$; Table 4$)$. The most frequent consultant specialties for surgical patients were cardiology $(8.7 \%, \mathrm{n}$ 64), hospital medicine $(8.7 \%, \mathrm{n}=64)$, and interventional cardiology $(4.2 \%, \mathrm{n}=31)$. Diagnostic radiology and anesthesiology were counted as consultant specialties during chart abstraction and represented large frequencies of consultants for both medical and surgical patients; however, these specialties were included in the "All Other" category because these physicians are inherent to the inpatient experience and are not considered consultants in the traditional sense (Table 4).

\section{KANSAS JOURNAL of MEDICINE}

\section{PATIENT HANDOFFS}

continued.
Table 4. Consulting specialty by service.

\begin{tabular}{|c|c|c|}
\hline Type of service & Frequency & Percent \\
\hline \multicolumn{3}{|l|}{ Medical } \\
\hline Nephrology & 20 & $9.2 \%$ \\
\hline Cardiology & 18 & $8.3 \%$ \\
\hline Pulmonary Disease & 11 & $5.0 \%$ \\
\hline General Surgery & 10 & $4.6 \%$ \\
\hline Infectious Diseases & 10 & $4.6 \%$ \\
\hline Cardiology Electrophysiology & 9 & $4.1 \%$ \\
\hline Interventional Cardiology & 9 & $4.1 \%$ \\
\hline Neurology & 8 & $3.7 \%$ \\
\hline All other & 113 & $56.4 \%$ \\
\hline \multicolumn{3}{|l|}{ Surgical } \\
\hline Cardiology & 64 & $8.7 \%$ \\
\hline Hospitalist & 64 & $8.7 \%$ \\
\hline Interventional Cardiology & 31 & $4.2 \%$ \\
\hline Pathology & 21 & $2.9 \%$ \\
\hline Pulmonary Disease & 16 & $2.2 \%$ \\
\hline All other & 491 & $73.2 \%$ \\
\hline
\end{tabular}

Resident physicians accounted for $14.3 \%(\mathrm{n}=291)$ of the total providers, and of the advanced practice professional providers, nurse practitioners had the most patient encounters $(16.4 \%, \mathrm{n}=333$; Table 2). For all providers, including attending physicians, consulting physicians, resident physicians, physician assistants, and nurse practitioners, the most represented specialties were anesthesiology $(22.3 \%, \mathrm{n}=453)$, diagnostic radiology $(17.6 \%, \mathrm{n}=358)$, general surgery $(12.2 \%, \mathrm{n}=247)$, and hospital medicine $(10.7 \%, \mathrm{n}=217)$.

For all inpatients, an average of two $(\mathrm{SD}=1.0)$ attending physicians participated in care over the duration of their stay with a range of one to six attending physicians. There was an average of one ( $\mathrm{SD}=1.0)$ handoff between attending physicians. The mean duration of an attending physician encounter was 94.5 hours $(\mathrm{SD}=90.0)$ for all inpatients. Patients in the study had an average of five ( $\mathrm{SD}=4.4)$ consulting physicians, two $(\mathrm{SD}=2.0)$ resident physicians, two $(\mathrm{SD}=0.9)$ physician assistants, and two $(\mathrm{SD}=1.7)$ nurse practitioners during a stay. There was an average of 10 total providers $(\mathrm{SD}=6.6)$, with a range of 1 to 46 total providers participating in care.

\section{DISCUSSION}

Patients admitted to a midwestern tertiary medical center with approximately 400 inpatient beds had an average of 10 healthcare providers. The patient population's demographics were comparable to both state and national inpatient demographics from the Healthcare Cost and Utilization Project (HCUP), a federal aggregation of state inpatient data. ${ }^{8}$ The majority of the current study population was female (52\%), which was similar to both national (56.7\%) and state (58.3\%) HCUP inpatient data and paralleled estimated 2019 census information for Sedgwick County, Kansas where approximately half 
KANSAS JOURNAL of MEDICINE PATIENT HANDOFFS

continued.

(50.6\%) of the population was female. ${ }^{9}$ However, the current study reported a mean age of 61 years, whereas national and state HCUP inpatient data reported 49 years as the mean age. ${ }^{8}$ This difference in age could be attributed to the timeframe of the study; the sample included patients hospitalized from November through January. Hospital admissions increase during the winter months, with the largest increase seen in patients 65 years or older. ${ }^{10}$ The difference in the average age of patients also could relate to insurance. Most individuals meet their insurance deductibles by the end of the calendar year, and some choose to undergo non-emergent elective procedures during this time. As the main consumers of elective orthopedic procedures are the elderly, ${ }^{11}$ this could explain the shift in the current study's demographics, especially considering that most patients were admitted to a surgical service.

The two most frequent MDCs under which patients were admitted were "Diseases and Disorders of the Musculoskeletal System and Connective Tissue" and "Diseases and Disorders of the Digestive System". Most of these MDCs included DRGs for surgical procedures. This result was concordant with 2016 national and state HCUP data, which ranked "Diseases and Disorders of the Musculoskeletal System and Connective Tissue" as the fourth most frequent MDC and "Diseases and Disorders of the Digestive System" as the sixth most frequent MDC for discharged patients. ${ }^{8}$

In the current study, the mean length of stay (LOS) was four days. This was slightly less than the mean LOS from national and state HCUP data reported in 2016 (4.6 and 4.3 days, respectively), as well as the American Hospital Association's data from 2018 (4.9 days).,12 This difference could be explained by the high volume of patients admitted to the hospital for elective surgery. In addition to the most common MDC, "Diseases and Disorders of the Musculoskeletal System and Connective Tissue" (the majority of which include surgical procedures), another common MDC in the current study, "Endocrine, Nutritional \& Metabolic Diseases \& Disorders", includes procedures for the treatment of obesity and laparoscopic gastric bypass surgeries, which account for most bariatric procedures and have a mean LOS of two days. ${ }^{13}$ Although mean LOS data vary for orthopedic- and abdominal-related elective surgeries, patients in high income countries who are admitted for these procedures have better outcomes and fewer postoperative complications, ${ }^{11,14-17}$ which could decrease the amount of time needed for inpatient postoperative recovery.

Co-management of surgical patients, where hospitalists and surgeons partner in the leadership of inpatient care during the perioperative period, was demonstrated in the current study. Aside from anesthesiology and diagnostic radiology, hospitalist medicine was one of the most frequent consultant physician specialties for patients admitted to the surgical service. This style of patient management has been studied frequently in the setting of orthopedic and neurosurgery with varying results; ${ }^{18}$ however, a 2015 study reported that co-management in orthopedic and neurosurgical patients significantly decreased the proportion of patients with at least one postoperative medical com- plication. ${ }^{19}$ Another study reported a decrease in one-year mortality in geriatric patients with hip fractures who were co-managed in an "orthogeriatric" team. These patients also showed better functional outcomes and a decreased risk of additional fractures. ${ }^{20}$ When taking into account that the majority of the patients in the current study were surgical patients undergoing orthopedic procedures, it is understandable that hospitalists would participate frequently in patient care to maximize patient outcomes.

The current study suggested there was an average of two attending physicians per patient stay, with one handoff between them. This result was concordant with similar research. In one study, inpatients admitted for pneumonia and heart failure had, on average, 2.05 and 1.78 hospitalists per stay, respectively. ${ }^{1}$ A 2016 study of patients with hospital stays longer than 21 days reported a mean number of attending and consulting physicians during the entire stay of 4.5 and 7.3, respectively. ${ }^{6}$

Implications and Future Research. This quantification of attending physicians, attending encounter time, total providers, and number of handoffs for inpatients' stays is a critical quality improvement and research metric. When more providers participate in patient care, length of inpatients' stays are longer, and patients' satisfaction with physician-to-patient communication is poorer. ${ }^{1,6}$ Identification of these variables is a foundational step in the improvement of healthcare delivery and patient satisfaction. For example, one study indicated that a relatively simple intervention of scheduling emergency department providers in overlapping shifts resulted in a $25 \%$ reduction in patient hand-offs, a decrease in 72-hour patient readmissions, improved charting times, and improved provider satisfaction. ${ }^{4}$

This study functions as a starting point for further research into quantifiable provider data and the impact of this data on inpatients' experience. As national healthcare spending is projected to grow to $20 \%$ of the total national gross domestic product by the year $2025,{ }^{21}$ careful analysis of resources used during an inpatient stay could help to control costs. Future research could investigate the number and types of providers participating in the care of patients admitted for specific MDCs and/or DRG; it also could hone the focus on specific outcomes like expenditures or patient mortality. Other future research could identify if communication gaps between providers exist, the effect(s) of these gaps on inpatients' care and indicate where process improvements could be initiated to maximize both inpatients' outcomes and the use of healthcare resources.

Limitations. There are several limitations that must be acknowledged. First, more medically complex patients inherently will require longer lengths of stay, more providers, and more handoffs. This inherent limitation to the study may have influenced the statistical interpretation of the data. Another limitation arises when considering that aggregate data for both medical and surgical patients were analyzed as one group. Again, because these groups may have inherent differences in the composition of their care teams and the amount/type of care needed, this may have skewed certain results such as number of attending physicians and total providers, length of stay, and attending encounter duration. Future studies can improve these limitations by stratifying patients per admission service (medical or surgical) or by analyzing specific DRGs/ MDCs. 


\section{CONCLUSIONS}

Understanding the quantitative provider data surrounding an inpatient stay is a foundational step in assessing the quality of the provider-inpatient encounter and potential areas for improvement. In this study, the average number of attending physicians and handoffs were reasonable; however, the total number of providers (including attending physicians, consulting physicians, resident physicians, and advanced practice professionals) involved in care were relatively high. Assessment of staffing and scheduling requirements by hospital administration could identify areas of improvement to reduce the potential for medical error caused by multiple providers being involved in patient care.

\section{REFERENCES}

${ }^{1}$ Epstein K, Juarez E, Epstein A, Loya K, Singer A. The impact of fragmentation of hospitalist care on length of stay. J Hosp Med 2010; 5(6):335-338. PMID: 20803671.

2 Traub O. Hospital Care Team Members. https://www.merckmanuals. com/home/special-subjects/hospital-care/hospital-care-team-members\#. 2018. Accessed June 26, 2020.

${ }^{3}$ Colvin MO, Eisen LA, Gong MN. Improving the patient handoff process in the intensive care unit: Keys to reducing errors and improving outcomes. Semin Respir Crit Care Med 2016; 37(1):96-106. PMID: 26820277.

${ }^{4}$ Yoshida H, Rutman LE, Chen J, et al. Waterfalls and handoffs: A novel physician staffing model to decrease handoffs in a pediatric emergency department. Ann Emerg Med 2019; 73(3):248-254. PMID: 30287122.

${ }^{5}$ Cheung DS, Kelly JJ, Beach C, et al. Improving handoffs in the emergency department. Ann Emerg Med 2010; 55(2):171-180. PMID: 19800711.

6 Schmocker RK, Holden SE, Vang X, et al. The number of inpatient consultations is negatively correlated with patient satisfaction in patients with prolonged hospital stays. Am J Surg 2016; 212(2):282-288. PMID: 26792276.

${ }^{7}$ Harris PA, Taylor R, Thielke R, Payne J, Gonzalez N, Conde JG. Research Electronic Data Capture (REDCap) - A metadata-driven methodology and workflow process for providing translational research informatics support. J Biomed Inform 2009; 42(2):377-381. PMID: 18929686.

${ }^{8}$ U.S. Agency for Healthcare Research and Quality. Healthcare Cost and Utilization Project. https://hcupnet.ahrq.gov/\#setup. Accessed July 26, 2020.

9 U.S. Census Bureau. Quick Facts: Sedgwick County, Kansas; Kansas. https://www.census.gov/quickfacts/fact/table/sedgwickcountykansas,KS/ PST045219. 2019. Accessed July 10, 2020.

${ }^{10}$ Patterson S. Do hospital admission rates increase in colder winters? A decadal analysis from an eastern county in England. J Public Health (Oxf) 2018; 40(2):221-228. PMID: 29106572.

${ }^{11}$ McDermott K, Freeman WJ, Elixhauser A. Overview of Operating Room Procedures During Inpatient Stays in U.S. Hospitals, 2014: Statistical Brief \#233. 2017. In: Healthcare Cost and Utilization Project (HCUP) Statistical Briefs [Internet]. Rockville (MD): Agency for Healthcare Research and Quality (US), 2006. https://www.ncbi.nlm.nih.gov/books/NBK487976/. Accessed February 9, 2021.

12 American Hospital Association. AHA Hospital Statistics. https://guide. prod.iam.aha.org/stats/states. 2021. Accessed July 25, 2020.

${ }_{13}$ Baker MT, Lara MD, Larson CJ, Lambert PJ, Mathiason MA, Kothari SN. Length of stay and impact on readmission rates after laparoscopic gastric bypass. Surg Obes Relat Dis 2006; 2(4):435-439. PMID: 16925375.

${ }_{14}$ Moghadamyeghaneh Z, Carmichael JC, Smith BR, et al. A comparison of outcomes of emergent, urgent, and elective surgical treatment of diverticulitis. Am J Surg 2015; 210(5):838-845. PMID: 26116319.

${ }^{15}$ International Surgical Outcomes Study Group. Global patient outcomes after elective surgery: Prospective cohort study in 27 low-, middle- and highincome countries. Br J Anaesth 2016; 117(5):601-609. PMID: 27799174.

${ }^{16}$ Le Manach Y, Collins G, Bhandari M, et al. Outcomes after hip fracture surgery compared with elective total hip replacement. JAMA 2015; 314(11):1159-1166. PMID: 26372585.

17 Beverly A, Brovman EY, Urman RD. Comparison of postoperative outcomes in elderly patients with a do-not-resuscitate order undergoing elective and nonelective hip surgery. Geriatr Orthop Surg Rehabil 2017; 8(2):78-86. PMID: 28540112.
KANSAS JOURNAL of MEDICINE

PATIENT HANDOFFS

continued.

${ }_{18}$ Auerbach AD, Wachter RM, Cheng HQ, et al. Comanagement of surgical patients between neurosurgeons and hospitalists. Arch Intern Med 2010; 170(22):2004-2010. PMID: 21149758.

19 Rohatgi N, Loftus P, Grujic O, Cullen M, Hopkins J, Ahuja N. Surgical comanagement by hospitalists improves patient outcomes: A propensity score analysis. Ann Surg 2016; 264(2):275-282. PMID: 26764873.

${ }^{20}$ Gosch M, Hoffmann-Weltin Y, Roth T, Blauth M, Nicholas JA, Kammerlander C. Orthogeriatric co-management improves the outcome of long-term care residents with fragility fractures. Arch Orthop Trauma Surg 2016; 136(10):1403-1409. PMID: 27501701.

${ }^{21}$ U.S. Centers for Medicare and Medicaid Services. National Health Expenditure Data. https://www.cms.gov/research-statistics-data-and-systems/ statistics-trends-and-reports/nationalhealthexpenddata. 2019. Accessed July 27, 2020.

Keywords: patient handoff, health care providers, inpatients, tertiary healthcare 